Annals of Pure and Applied Mathematics

Vol. 21, No. 2, 2020, 149-159

ISSN: 2279-087X (P), 2279-0888(online)

Published on 21 June 2020

Annals of

www.researchmathsci.org

DOI: http://dx.doi.org/10.22457/apam.v21n2a11671

Pure and Applied

Mathematics

\title{
Strong Domination Parameters in Operations on Fuzzy Graphs
}

\author{
O.T.Manjusha \\ Department of Mathematics \\ Kerala Govt. Polytechnic College, Westhill, Calicut \\ Kozhikode-673005, India \\ E-mail: manjushaot@gmail.com
}

Received 28 May 2020; accepted 12 June 2020

\begin{abstract}
In this paper, the concept of domination is introduced on various operations on fuzzy graphs such as union, join, composition and cartesian product using strong arcs. The different types of strong domination parameters of union and join of two fuzzy graphs is obtained.It is obtained the strong domination set of cartesian product of two fuzzy graphs.
\end{abstract}

Keywords: Fuzzy graph, strong arcs, union, join, composition cartesian product, fuzzy tree, fuzzy cycles.

AMS Mathematics Subject Classification (2010): $05 \mathrm{C} 69$

\section{Introduction}

Fuzzy graphs were introduced by Rosenfeld, who has described the fuzzy analogue of several graph theoretic concepts like paths, cycles, trees and connectedness [19]. Bhutani and Rosenfeld have introduced the concept of strong arcs [2].

The work on fuzzy graphs was also done by Mordeson, Pradip, Talebi,Yeh, Michael, sunil and Pathinathan [13, 18, 29, 30, 31, 32, 33]. It was during 1850s, a study of dominating sets in graphs started purely as a problem in the game of chess. Chess enthusiasts in Europe considered the problem of determining the minimum number of queens that can be placed on a chess board so that all the squares are either attacked by a queen or occupied by a queen. The concept of domination in graphs was introduced by Ore and Berge in 1962, the domination number and independent domination number are introduced by Cockayne and Hedetniemi [6]. Connected domination in graphs was discussed by Sampathkumar and Walikar [20]. Somasundaram and Somasundaram discussed domination in fuzzy graphs. They defined domination using effective edges in fuzzy graph [22, 23]. Nagoorgani and Chandrasekharan defined domination in fuzzy graphs using strong arcs [15]. Manjusha and Sunitha discussed some concepts in domination and total domination in fuzzy graphs using strong arcs $[7,8]$. In this paper, we study various operations on fuzzy graphs such as union,join, composition and cartesian product and study their domination parameters using strong arcs.

\section{Preliminaries}

We summarize briefly some basic definitions in fuzzy graphs which are presented in $[1,2$, 


\section{O.T.Manjusha}

$4,14,15,19,22,25]$.

A fuzzy graph is denoted by $G:(V, \sigma, \mu)$ where $V$ is a node set, $\sigma$ is a fuzzy subset of $V$ and $\mu$ is a fuzzy relation on $\sigma$. i.e., $\mu(x, y) \leq \sigma(x) \wedge \sigma(y)$ for all $x, y \in V$. We call $\sigma$ the fuzzy node set of $G$ and $\mu$ the fuzzy arc set of $G$, respectively. We consider fuzzy graph $G$ with no loops and assume that $V$ is finite and nonempty, $\mu$ is reflexive (i.e., $\mu(x, x)=\sigma(x)$, for all $x$ ) and symmetric (i.e., $\mu(x, y)=\mu(y, x)$, for all $(x, y))$. In all the examples $\sigma$ is chosen suitably. Also, we denote the underlying crisp graph by $G^{*}:\left(\sigma^{*}, \mu^{*}\right)$ where $\sigma^{*}=\{u \in V: \sigma(u)>0\} \quad$ and $\mu^{*}=\{(u, v) \in V \times$ $V: \mu(u, v)>0\}$. Throughout we assume that $\sigma^{*}=V$. The fuzzy graph $H:(\tau, v)$ is said to be a partial fuzzy subgraph of $G:(\sigma, \mu)$ if $v \subseteq \mu$ and $\tau \subseteq \sigma$. In particular we call $H:(\tau, v)$, a fuzzy subgraph of $G:(\sigma, \mu)$ if $\tau(u)=\sigma(u)$ for all $u \in \tau^{*}$ and $v(u, v)=$ $\mu(u, v)$ for all $(u, v) \in v^{*}$. A fuzzy subgraph $H:(\tau, v)$ spans the fuzzy graph $G:(V, \sigma, \mu)$ if $\tau=\sigma$. The fuzzy graph $H:(P, \tau, v)$ is called an induced fuzzy subgraph of $G:(V, \sigma, \mu)$ induced by $P$ if $P \subseteq V$ and $\tau(u)=\sigma(u)$ for all $u \in P$ and $v(u, v)=\mu(u, v)$ for all $u, v \in P$. We shall use the notation $\langle P\rangle$ to denote the fuzzy subgraph induced by $P$. $G:(V, \sigma, \mu)$ is called trivial if $\left|\sigma^{*}\right|=1$.

In a fuzzy graph $G:(V, \sigma, \mu)$, a path $P$ of length $n$ is a sequence of distinct nodes $u_{0}, u_{1}, \ldots, u_{n}$ such that $\mu\left(u_{i-1}, u_{i}\right)>0, i=1,2, \ldots, n$ and the degree of membership of a weakest arc is defined as its strength. If $u_{0}=u_{n}$ and $n \geq 3$ then $P$ is called a cycle and $P$ is called a fuzzy cycle, if it contains more than one weakest arc. The strength of a cycle is the strength of the weakest arc in it. The strength of connectedness between two nodes $x$ and $y$ is defined as the maximum of the strengths of all paths between $x$ and $y$ and is denoted by $\operatorname{CONN}_{G}(x, y)$.

A fuzzy graph $G:(\sigma, \mu)$ is connected if for every $x, y$ in $\sigma^{*}, \operatorname{CONN}_{G}(x, y)>0$. An $\operatorname{arc}(u, v)$ of a fuzzy graph is called an effective arc (M-strong arc) if $\mu(u, v)=$ $\sigma(u) \wedge \sigma(v)$. Then $u$ and $v$ are called effective neighbors. The set of all effective neighbors of $u$ is called effective neighborhood of $u$ and is denoted by $E N(u)$.

A fuzzy graph $G$ is said to be complete if $\mu(u, v)=\sigma(u) \wedge \sigma(v)$, for all $u, v \in \sigma^{*}$ and is denoted by $K_{\sigma}$.

The order $p$ and size $q$ of a fuzzy graph $G:(\sigma, \mu)$ are defined to be $p=$ $\sum_{x \in V} \sigma(x)$ and $q=\sum_{(x, y) \in V \times V} \mu(x, y)$.

Let $G:(V, \sigma, \mu)$ be a fuzzy graph and $S \subseteq V$. Then the scalar cardinality of $S$ is defined to be $\sum_{v \in S} \sigma(v)$ and it is denoted by $|S|$. Let $p$ denotes the scalar cardinality of $V$, also called the order of $G$.

An arc of a fuzzy graph is called strong if its weight is at least as great as the strength of connectedness of its end nodes when it is deleted. Depending on $\operatorname{CONN}_{G}(x, y)$ of an arc $(x, y)$ in a fuzzy graph $G$, Sunil Mathew and Sunitha [25] defined three different types of arcs. Note that $\operatorname{CONN}_{G-(x, y)}(x, y)$ is the the strength of connectedness between $x$ and $y$ in the fuzzy graph obtained from $G$ by deleting the $\operatorname{arc}(x, y)$. An $\operatorname{arc}(x, y)$ in $G$ is $\boldsymbol{\alpha}$ - strong if $\mu(x, y)>\operatorname{CONN}_{G-(x, y)}(x, y)$. An arc $(x, y)$ in $G$ is $\boldsymbol{\beta}$ - strong if $\mu(x, y)=\operatorname{CONN}_{G-(x, y)}(x, y)$. An $\operatorname{arc}(x, y)$ in $G$ is $\boldsymbol{\delta}-\operatorname{arc}$ if $\mu(x, y)<\operatorname{CONN}_{G-(x, y)}(x, y)$.

Thus an arc $(x, y)$ is a strong arc if it is either $\alpha-$ strong or $\beta-$ strong. A path $P$ is called strong path if $P$ contains only strong arcs.

A fuzzy graph $G$ is said to be bipartite [22] if the node set $V$ can be partitioned 
Strong Domination Parameters in Operations on Fuzzy Graphs

into two non empty sets $V_{1}$ and $V_{2}$ such that $\mu\left(v_{1}, v_{2}\right)=0$ if $v_{1}, v_{2} \in V_{1}$ or $v_{1}, v_{2} \in$ $V_{2}$. Further if $\mu(u, v)=\sigma(u) \wedge \sigma(v)$ for all $u \in V_{1}$ and $v \in V_{2}$ then $G$ is called a complete bipartite graph and is denoted by $K_{\sigma_{1}, \sigma_{2}}$, where $\sigma_{1}$ and $\sigma_{2}$ are respectively the restrictions of $\sigma$ to $V_{1}$ and $V_{2}$.

A connected fuzzy graph $G=(V, \sigma, \mu)$ is called a fuzzy tree if it has a fuzzy spanning subgraph $F:(\sigma, v)$, which is a tree [spanning tree], where for all arcs $(x, y)$ not in $F$ there exists a path from $x$ to $y$ in $F$ whose strength is more than $\mu(x, y)$ [19]. Note that here $F$ is a tree which contains all nodes of $G$ and hence is a spanning tree of $G$.

A maximum spanning tree of a connected fuzzy graph $G:(V, \sigma, \mu)$ is a fuzzy spanning subgraph $T:(\sigma, v)$, such that $T$ is a tree, and for which $\sum_{u \neq v} v(u, v)$ is maximum. A node which is not an endnode of $T$ is called an internal node of $T$ [14].

A node $u$ is said to be isolated if $\mu(u, v)=0$ for all $v \neq u$.

\section{Strong domination parametrs in operations on fuzzy graphs}

The concept of domination in operations on fuzzy graphs was introduced by Somasundaram [24]. The author used effective arcs in this concept. This study leads to group all the fuzzy graphs without effective arcs under one category. Also note that each effective arc is strong, but a strong arc need not be effective [2]. Hence a generalized study of domination parameters in operations on fuzzy graphs using strong arcs is more relevant. Let us recall some basic definitions of domination parameters and operations on fuzzy graphs.

Definition 1. [15] A node $v$ in a fuzzy graph $G$ is said to strongly dominate itself and each of its strong neighbors, i.e., $v$ strongly dominates the nodes in $\mathrm{N}_{s}[\mathrm{v}]$. A set $\mathrm{D}$ of nodes of $G$ is a strong dominating set of $G$ if every node of $V(G)-D$ is a strong neighbor of some node in D.

Definition 2. [9] The weight of a strong dominating set $D$ is defined as $W(D)=$ $\sum_{u \in D} \mu(u, v)$, where $\mu(u, v)$ is the minimum of the membership values(weights) of strong arcs incident on $u$. The strong domination number of a fuzzy graph $G$ is defined as the minimum weight of strong dominating sets of $G$ and it is denoted by $\gamma_{s}(G)$ or simply $\gamma_{s}$. A minimum strong dominating set in a fuzzy graph $G$ is a strong dominating set of minimum weight.

Definition 3. [8] A set D of nodes in a fuzzy graph $G:(V, \sigma, \mu)$ is a strong total(open) dominating set of $G$ if every node of $G$ is a strong neighbor of at least one node of $D$.

Definition 4. [8] The weight of a strong total dominating set $D$ is defined as $W(D)=$ $\sum_{u \in D} \mu(u, v)$, where $\mu(u, v)$ is the minimum of the weight of the strong arcs incident on $u$. The strong total domination number of a fuzzy graph $G$ is defined as the minimum weight of strong total dominating sets of $G$ and it is denoted by $\gamma_{s t}(G)$ or simply $\gamma_{s t}$. A minimum strong total dominating set in a fuzzy graph $G$ is a strong total dominating set of minimum weight.

Definition 5. [10] A strong dominating set D of a fuzzy graph G: $(V, \sigma, \mu)$ is a strong connected dominating set of $\mathrm{G}$ if the induced fuzzy subgraph $\langle D\rangle$ is connected. 


\section{O.T.Manjusha}

Definition 6. [10] The weight of a strong connected dominating set $D$ is defined as $\mathrm{W}(\mathrm{D})=\sum_{\mathrm{u} \in \mathrm{D}} \mu(\mathrm{u}, \mathrm{v})$, where $\mu(\mathrm{u}, \mathrm{v})$ is the minimum of the membership values(weights) of strong arcs incident on $u$. The strong connected domination number of a fuzzy graph $G$ is defined as the minimum weight of strong connected dominating sets of $G$ and it is denoted by $\gamma_{\mathrm{sc}}(\mathrm{G})$ or simply $\gamma_{\mathrm{sc}}$. A minimum strong connected dominating set in a fuzzy graph $\mathrm{G}$ is a strong connected dominating set of minimum weight.

Definition 7. [15] Two nodes in a fuzzy graph $G:(V, \sigma, \mu)$ are said to be strongly independent if there is no strong arc between them. A set of nodes in $G$ is strong independent if any two nodes in the set are strongly independent.

Definition 8. [15] A strong dominating set D of a fuzzy graph $G:(V, \sigma, \mu)$ is called a strong independent dominating set if $\mathrm{D}$ is strongly independent.

Definition 9. The weight of a strong independent dominating set $D$ is defined as $\mathrm{W}(\mathrm{D})=\sum_{\mathrm{u} \in \mathrm{D}} \mu(\mathrm{u}, \mathrm{v})$, where $\mu(\mathrm{u}, \mathrm{v})$ is the minimum of the membership values(weights) of the strong arcs incident on $u$. The strong independent domination number of a fuzzy graph $G$ is defined as the minimum weight of strong independent dominating sets of $G$ and it is denoted by $S_{i}(G)$ or simply $S_{i}$. A minimum strong independent dominating set in a fuzzy graph $\mathrm{G}$ is a strong independent dominating set of minimum weight.

Definition 10. [17] Let $G:(V, \sigma, \mu)$ be a fuzzy graph. A set $M$ of strong arcs in $G$ such that no two arcs in $M$ have a common node is called a strong independent set of arcs or a strong matching in $\mathrm{G}$.

Definition 11. [12] Let $M$ be a strong matching in a fuzzy graph $G:(V, \sigma, \mu)$. If e = $(u, v) \in M$, then we say that $M$ strongly matches $u$ to $v$. The weight of a strong matching is defined as $\mathrm{W}(\mathrm{M})=\sum_{(\mathrm{u}, \mathrm{v}) \in \mathrm{M}} \mu(\mathrm{u}, \mathrm{v})$. The strong arc independence number or strong matching number of a fuzzy graph $G$ is defined as the maximum weight of strong matchings of $G$ and it is denoted by $\beta_{\mathrm{S}_{1}}(\mathrm{G})$ or simply $\beta_{\mathrm{S}_{1}}$. A maximum strong matching in a fuzzy graph $G$ is a strong matching of maximum weight.

Definition 12. [12] Let $G:(V, \sigma, \mu)$ be a fuzzy graph and let $M$ be a strong matching in $G$. Then $M$ is called perfect strong matching if $M$ strongly matches every node of $G$ to some node of $\mathrm{G}$.

Definition 13. [12] Let $G:(V, \sigma, \mu)$ be a fuzzy graph. A set $D \subseteq V$ of nodes is said to be a strong paired dominating set if $\mathrm{D}$ is a strong dominating set and the induced fuzzy subgraph $\langle D\rangle$ has a perfect strong matching. The weight of a strong paired dominating set is defined as $W(D)=\sum_{u \in D} \mu(u, v)$, where $\mu(u, v)$ is the minimum of the weights of strong arcs incident on $u$. The strong paired domination number of a fuzzy graph $\mathrm{G}$ is defined as the minimum weight of strong paired dominating sets of $\mathrm{G}$ and it is denoted by $\gamma_{\mathrm{spr}}(\mathrm{G})$ or simply $\gamma_{\mathrm{spr}}$. A minimum strong paired dominating set in a fuzzy graph $G$ is a strong paired dominating set of minimum weight. 
Strong Domination Parameters in Operations on Fuzzy Graphs

Definition 14. $[13,14]$ Union of two fuzzy graphs: Let $G_{1}:\left(\sigma_{1}, \mu_{1}\right)$ and $G_{2}:\left(\sigma_{2}, \mu_{2}\right)$ be two fuzzy graphs with $\mathrm{G}_{1}^{*}:\left(\mathrm{V}_{1}, \mathrm{E}_{1}\right)$ and $\mathrm{G}_{2}^{*}:\left(\mathrm{V}_{2}, \mathrm{E}_{2}\right)$ with $\mathrm{V}_{1} \cap \mathrm{V}_{2}=\phi$ and let $G^{*}=$ $G_{1}^{*} \cup G_{2}^{*}=\left(V_{1} \cup V_{2}, E_{1} \cup E_{2}\right)$ be the union of $G_{1}^{*}$ and $G_{2}^{*}$. Then the union of two fuzzy graphs $G_{1}$ and $G_{2}$ is a fuzzy graph $G:\left(\sigma_{1} \cup \sigma_{2}, \mu_{1} \cup \mu_{2}\right)$ defined by

and

$$
\left(\sigma_{1} \cup \sigma_{2}\right)(u)= \begin{cases}\sigma_{1}(u) & \text { if } u \in V_{1} \backslash V_{2} \\ \sigma_{2}(u) & \text { if } u \in V_{2} \backslash V_{1}\end{cases}
$$

$$
\left(\mu_{1} \cup \mu_{2}\right)(u, v)= \begin{cases}\mu_{1}(u, v) & \text { if }(u, v) \in E_{1} \backslash E_{2} \\ \mu_{2}(u, v) & \text { if }(u, v) \in E_{2} \backslash E_{1}\end{cases}
$$

We now proceed to study various domination parameters in union of two fuzzy graphs.

Theorem 15. If $G_{1}$ and $G_{2}$ are two fuzzy fuzzy graphs on $V_{1}$ and $V_{2}$ respectively with $\mathrm{V}_{1} \cap \mathrm{V}_{2}=\phi$.Then

1. $\gamma_{s}\left(G_{1} \cup G_{2}\right)=\gamma_{s}\left(G_{1}\right)+\gamma_{s}\left(G_{2}\right)$

2. If $G_{1}$ and $G_{2}$ are fuzzy graphs having no isolated nodes, then

3. $S_{i}\left(G_{1} \cup G_{2}\right)=S_{i}\left(G_{1}\right)+S_{i}\left(G_{2}\right)$

$$
\gamma_{s t}\left(G_{1} \cup G_{2}\right)=\gamma_{s t}\left(G_{1}\right)+\gamma_{s t}\left(G_{2}\right)
$$

4. If $G_{1}$ and $G_{2}$ are fuzzy graphs having no isolated nodes, then

$$
\gamma_{s p r}\left(G_{1} \cup G_{2}\right)=\gamma_{s p r}\left(G_{1}\right)+\gamma_{s p r}\left(G_{2}\right)
$$

Proof: Any type strong dominating set $D$ of $G_{1} \cup G_{2}$ is of the form $D=D_{1} \cup D_{2}$, where $D_{1}$ is any type strong dominating set of $G_{1}$ and $D_{2}$ is any type strong dominating set of $G_{2}$ It follows that results $1,2,3$ and 4 hold.

Remark 16. Since $G_{1} \cup G_{2}$ is disconnected $\gamma_{s c}\left(G_{1} \cup G_{2}\right)$ is not defined.

Definition 17. $[13,14]$ Join of two fuzzy graphs:Let $G_{1}:\left(\sigma_{1}, \mu_{1}\right)$ and $G_{2}:\left(\sigma_{2}, \mu_{2}\right)$ be two fuzzy graphs with $G_{1}^{*}:\left(V_{1}, E_{1}\right)$ and $G_{2}^{*}:\left(V_{2}, E_{2}\right)$ with $V_{1} \cap V_{2}=\phi$ Consider the join $G^{*}=G_{1}^{*}+G_{2}^{*}=\left(V_{1} \cup V_{2}, E_{1} \cup E_{2} \cup E^{\prime}\right)$ of graphs where $E^{\prime}$ is the set of all arcs joining the nodes of $V_{1}$ and $V_{2}$ where we assume that $V_{1} \cap V_{2}=\phi$. Then the join of two fuzzy graphs $G_{1}$ and $G_{2}$ is a fuzzy graph $G=G_{1}+G_{2}:\left(\sigma_{1}+\sigma_{2}, \mu_{1}+\mu_{2}\right)$ defined by $\left(\sigma_{1}+\sigma_{2}\right)(u)=\left(\sigma_{1} \cup \sigma_{2}\right)(u), u \in V_{1} \cup V_{2}$

and

$$
\left(\mu_{1}+\mu_{2}\right)(u, v)= \begin{cases}\left(\mu_{1} \cup \mu_{2}\right)(u, v) & \text { if }(u, v) \in E_{1} \cup E_{2} \text { and } \\ \sigma_{1}(u) \wedge \sigma_{2}(v) & \text { if }(u, v) \in E^{\prime}\end{cases}
$$

We now proceed to study various domination parameters in join of two fuzzy graphs.

Theorem 18. If $G_{1}$ and $G_{2}$ are two fuzzy fuzzy graphs on $V_{1}$ and $V_{2}$ respectively with $V_{1} \cap V_{2}=\phi$.Then $\gamma_{s}\left(G_{1}+G_{2}\right)=\wedge \quad\left\{W(D), W(\{u, v\}): u \in V_{1}, v \in V_{2}\right\}$, where $D$ is any minimal strong dominating set of $G_{1}$ or $G_{2}$ which is also a minimal strong dominating set of $G_{1}+G_{2}$.

Proof: From the definition of $G_{1}+G_{2}$ it is clear that any arc of the form $(u, v)$, where $u \in V_{1}$ and $v \in V_{2}$ is a strong arc since it is an effective arc. Hence any node of $V_{1}$ 


\section{O.T.Manjusha}

strongly dominates all the nodes of $V_{2}$ and vice-versa. Now, let $D$ be any minimal strong dominating set of $G_{1}+G_{2}$. Then $D$ has the following 3 possibilities.

1. $D=D_{1}$ where $D_{1}$ is a minimal strong dominating set of $G_{1}$

2. $D=D_{2}$ where $D_{2}$ is a minimal strong dominating set of $G_{2}$

3. $D=\{u, v\}$ where $u \in V_{1}, v \in V_{2},\{u\}$ is not a strong dominating set of $G_{1}$ and $\{v\}$ is not a strong dominating set of $G_{2}$. Hence

$$
\gamma_{s}\left(G_{1}+G_{2}\right)=\wedge \quad\left\{W(D), W(\{u, v\}): u \in V_{1}, v \in V_{2}\right\} .
$$

Theorem 19. If $G_{1}$ and $G_{2}$ are two fuzzy fuzzy graphs on $V_{1}$ and $V_{2}$ respectively with $\mathrm{V}_{1} \cap \mathrm{V}_{2}=\phi$.Then

$$
S_{i}\left(G_{1}+G_{2}\right)=\wedge \quad\{W(D)\}
$$

where $D$ is any minimal strong independent dominating set of $G_{1}$ or $G_{2}$ which is also a minimal strong independent dominating set of $G_{1}+G_{2}$.

Proof: Since any node of $V_{1}$ strongly dominates every node of $V_{2}$ in $G_{1}+G_{2}$, any strong independent set in $G_{1}+G_{2}$ is either a subset of $V_{1}$ or a subset of $V_{2}$. Hence any minimal strong independent dominating set $D$ of $G_{1}+G_{2}$ is one of the following forms.

1. $D=D_{1}$, where $D_{1}$ is a minimal strong independent dominating set of $G_{1}$

2. $D=D_{2}$, where $D_{2}$ is a minimal strong independent dominating set of $G_{2}$

Hence $S_{i}\left(G_{1}+G_{2}\right)=\wedge\{W(D)\}$, where $D$ is any minimal strong independent dominating set of $G_{1}$ or $G_{2}$ which is also a minimal strong independent dominating set of $G_{1}+G_{2}$.

Theorem 20. If $G_{1}$ and $G_{2}$ are two fuzzy fuzzy graphs on $V_{1}$ and $V_{2}$ respectively with $\mathrm{V}_{1} \cap \mathrm{V}_{2}=\phi$.Then

1. If $G_{1}$ and $G_{2}$ has no isolated nodes, then

$$
\gamma_{s t}\left(G_{1}+G_{2}\right)=\wedge \quad\left\{W(D), W(\{u, v\}): u \in V_{1}, v \in V_{2}\right\}
$$

where $D$ is any minimal strong total dominating set of $G_{1}$ or $G_{2}$ which is also a minimal strong total dominating set of $G_{1}+G_{2}$.

2. If $G_{1}$ has no isolated nodes and $G_{2}$ has an isolated node, then

$$
\gamma_{s t}\left(G_{1}+G_{2}\right)=\wedge \quad\left\{W(D), W(\{u, v\}): u \in V_{1}, v \in V_{2}\right\}
$$

where $D$ is any minimal strong total dominating set of $G_{1}$ which is also a minimal strong total dominating set of $G_{1}+G_{2}$.

3.If $G_{1}$ has an isolated node and $G_{2}$ has no isolated nodes, then

$$
\gamma_{s t}\left(G_{1}+G_{2}\right)=\Lambda \quad\left\{W(D), W(\{u, v\}): u \in V_{1}, v \in V_{2}\right\}
$$

where $D$ is any minimal strong total dominating set of $G_{2}$ which is also a minimal strong total dominating set of $G_{1}+G_{2}$.

4. If both $G_{1}$ and $G_{2}$ have isolated nodes, then

$$
\gamma_{s t}\left(G_{1}+G_{2}\right)=\wedge \quad\left\{W(\{u, v\}): u \in V_{1}, v \in V_{2}\right\}
$$

Proof: Note that for any two fuzzy graphs $G_{1}$ and $G_{2}$, its join $G_{1}+G_{2}$ is a fuzzy graph having no isolated nodes and hence $\gamma_{s t}\left(G_{1}+G_{2}\right)$ always exists. We have the following cases.

CASE 1: Both $G_{1}$ and $G_{2}$ have no isolated nodes.

In this case $\gamma_{s t}\left(G_{1}\right)$ and $\gamma_{s t}\left(G_{2}\right)$ exist. Any minimal strong total dominating set $D$ of $G_{1}+G_{2}$ is one of the following forms.

1. $D=D_{1}$, where $D_{1}$ is a minimal strong total dominating set of $G_{1}$.

2. $D=D_{2}$, where $D_{2}$ is a minimal strong total dominating set of $G_{2}$. 
Strong Domination Parameters in Operations on Fuzzy Graphs

3. $D=\{u, v\}$, where $u \in V_{1}$ and $v \in V_{2}$.

Hence $\gamma_{s t}\left(G_{1}+G_{2}\right)=\wedge \quad\left\{W(D), W(\{u, v\}): u \in V_{1}, v \in V_{2}\right\}$

where $D$ is any minimal strong total dominating set of $G_{1}$ or $G_{2}$ which is also a minimal strong total dominating set of $G_{1}+G_{2}$.

CASE II: $G_{1}$ has no isolated nodes and $G_{2}$ has an isolated node.

In this case $\gamma_{s t}\left(G_{2}\right)$ does not exist. Hence any strong total dominating set of $G_{1}+G_{2}$ has nonempty intersection with $V_{1}$. Now, let $D$ be any minimal strong total dominating set of $G_{1}+G_{2}$. Then $D$ is one of the following forms:

1. $D=D_{1}$, where $D_{1}$ is a minimal strong total dominating set of $G_{1}$.

2. $D=\{u, v\}$, where $u \in V_{1}$ and $v \in V_{2}$.

Hence $\gamma_{s t}\left(G_{1}+G_{2}\right)=\wedge \quad\left\{W(D), W(\{u, v\}): u \in V_{1}, v \in V_{2}\right\}$

where $D$ is any minimal strong total dominating set of $G_{1}$ which is also a minimal strong total dominating set of $G_{1}+G_{2}$.

CASE III: $G_{1}$ has an isolated node and $G_{2}$ has no isolated nodes.

Proof is similar to that of CASE II.

CASE IV: Both $G_{1}$ and $G_{2}$ have isolated nodes.

In this case both $\gamma_{s t}\left(G_{1}\right)$ and $\gamma_{s t}\left(G_{2}\right)$ do not exist. Hence any strong total dominating set of $G_{1}+G_{2}$ has nonempty intersection with both $V_{1}$ and $V_{2}$, so that any minimal strong total dominating set of $G_{1}+G_{2}$ is of the form $\{u, v\}$, where $u \in V_{1}, v \in V_{2}$. Hence

$$
\gamma_{s t}\left(G_{1}+G_{2}\right)=\wedge \quad\left\{W(\{u, v\}): u \in V_{1}, v \in V_{2}\right\}
$$

Theorem 21. If $G_{1}$ and $G_{2}$ are two fuzzy fuzzy graphs on $V_{1}$ and $V_{2}$ respectively with $\mathrm{V}_{1} \cap \mathrm{V}_{2}=\phi$. Then

1. If $G_{1}$ and $G_{2}$ has no isolated nodes, then

$$
\gamma_{s p r}\left(G_{1}+G_{2}\right)=\wedge \quad\left\{W(D), W(\{u, v\}): u \in V_{1}, v \in V_{2}\right\}
$$

where $D$ is any minimal strong paired dominating set of $G_{1}$ or $G_{2}$ which is also a minimal strong paired dominating set of $G_{1}+G_{2}$.

2. If $G_{1}$ has no isolated nodes and $G_{2}$ has an isolated node, then

$$
\gamma_{s p r}\left(G_{1}+G_{2}\right)=\wedge\left\{W(D), W(\{u, v\}): u \in V_{1}, v \in V_{2}\right\}
$$

where $D$ is any minimal strong paired dominating set of $G_{1}$ which is also a minimal strong paired dominating set of $G_{1}+G_{2}$.

3.If $G_{1}$ has an isolated node and $G_{2}$ has no isolated nodes, then

$$
\gamma_{s p r}\left(G_{1}+G_{2}\right)=\Lambda \quad\left\{W(D), W(\{u, v\}): u \in V_{1}, v \in V_{2}\right\}
$$

where $D$ is any minimal strong paired dominating set of $G_{2}$ which is also a minimal strong paired dominating set of $G_{1}+G_{2}$.

4. If both $G_{1}$ and $G_{2}$ have isolated nodes, then

$$
\gamma_{s p r}\left(G_{1}+G_{2}\right)=\wedge \quad\left\{W(\{u, v\}): u \in V_{1}, v \in V_{2}\right\}
$$

Proof: Note that for any two fuzzy graphs $G_{1}$ and $G_{2}$, its join $G_{1}+G_{2}$ is a fuzzy graph having no isolated nodes and hence $\gamma_{s p r}\left(G_{1}+G_{2}\right)$ always exists. The proof of 1,2,3 and 4 of Theorem 21 is similar to that of Theorem 20 .

Theorem 22. If $G_{1}$ and $G_{2}$ are two fuzzy fuzzy graphs on $V_{1}$ and $V_{2}$ respectively with $\mathrm{V}_{1} \cap \mathrm{V}_{2}=\phi$.Then

1. If both $G_{1}$ and $G_{2}$ are connected, then 


\section{O.T.Manjusha}

$$
\gamma_{s c}\left(G_{1}+G_{2}\right)=\wedge \quad\left\{W(D), W(\{u, v\}): u \in V_{1}, v \in V_{2}\right\}
$$

where $D$ is any minimal strong connected dominating set of $G_{1}$ or $G_{2}$ which is also a minimal strong connected dominating set of $G_{1}+G_{2}$.

2. If $G_{1}$ is connected and $G_{2}$ is not connected, then

$$
\gamma_{s c}\left(G_{1}+G_{2}\right)=\wedge \quad\left\{W(D), W(\{u, v\}): u \in V_{1}, v \in V_{2}\right\}
$$

where $D$ is any minimal strong connected dominating set of $G_{1}$ which is also a minimal strong connected dominating set of $G_{1}+G_{2}$.

3.If $G_{1}$ is not connected and $G_{2}$ is connected, then

$$
\gamma_{s c}\left(G_{1}+G_{2}\right)=\wedge \quad\left\{W(D), W(\{u, v\}): u \in V_{1}, v \in V_{2}\right\}
$$

where $D$ is any minimal strong connected dominating set of $G_{2}$ which is also a minimal strong connetced dominating set of $G_{1}+G_{2}$.

4. If both $G_{1}$ and $G_{2}$ are disconnected, then

$$
\gamma_{s c}\left(G_{1}+G_{2}\right)=\Lambda \quad\left\{W(\{u, v\}): u \in V_{1}, v \in V_{2}\right\}
$$

Proof: Note that for any two fuzzy graphs $G_{1}$ and $G_{2}$, the fuzzy graph $G_{1}+G_{2}$ is connected and hence $\gamma_{s c}\left(G_{1}+G_{2}\right)$ always exists. The proof of 1,2,3 and 4 of Theorem 22 is similar to that of Theorem 20.

Definition 23. [13, 14] Cartesian product of two fuzzy graphs: Let $G_{1}:\left(\sigma_{1}, \mu_{1}\right)$ and $\mathrm{G}_{2}:\left(\sigma_{2}, \mu_{2}\right)$ be two fuzzy graphs with $\mathrm{G}_{1}^{*}:\left(\mathrm{V}_{1}, \mathrm{E}_{1}\right)$ and $\mathrm{G}_{2}^{*}:\left(\mathrm{V}_{2}, \mathrm{E}_{2}\right)$ with $\mathrm{V}_{1} \cap \mathrm{V}_{2}=\phi$. Consider the Cartesian product $G^{*}=G_{1}^{*} \times G_{2}^{*}=(V, E)$ where $V=V_{1} \times V_{2}$ and $E=\left\{\left(\left(u, u_{2}\right),\left(u, v_{2}\right)\right): u \in V_{1},\left(u_{2}, v_{2}\right) \in E_{2}\right\} \cup\left\{\left(\left(u_{1}, w\right),\left(v_{1}, w\right)\right):,\left(u_{1}, v_{1}\right) \in\right.$ $\left.E_{1}, w \in V_{2}\right\}$. Then the Cartesian product of two fuzzy graphs $G_{1}$ and $G_{2}$ is a fuzzy graph $G=G_{1} \times G_{2}:\left(\sigma_{1} \times \sigma_{2}, \mu_{1} \times \mu_{2}\right)$ defined by

$$
\left(\sigma_{1} \times \sigma_{2}\right)\left(u_{1}, u_{2}\right)=\left(\sigma_{1}\left(u_{1}\right) \wedge \sigma_{2}\right)\left(u_{2}\right), \forall\left(u_{1}, u_{2}\right) \in V
$$

and $\left(\mu_{1} \times \mu_{2}\right)\left(\left(u, u_{2}\right),\left(u, v_{2}\right)\right)=\sigma_{1}(u) \wedge \mu_{2}\left(u_{2}, v_{2}\right) \forall u \in V_{1},\left(u_{2}, v_{2}\right) \in E_{2}$

$$
\left(\mu_{1} \times \mu_{2}\right)\left(\left(u_{1}, w\right),\left(v_{1}, w\right)\right)=\sigma_{2}(w) \wedge \mu_{1}\left(u_{1}, v_{1}\right) \forall w \in V_{2},\left(u_{1}, v_{1}\right) \in E_{1}
$$

Definition 24. [13, 14] Composition of two fuzzy graphs: Let $G_{1}:\left(\sigma_{1}, \mu_{1}\right)$ and $G_{2}:\left(\sigma_{2}, \mu_{2}\right)$ be two fuzzy graphs with $G_{1}^{*}:\left(V_{1}, E_{1}\right)$ and $G_{2}^{*}:\left(V_{2}, E_{2}\right)$ with $V_{1} \cap V_{2}=\phi$. Consider the composition $G^{*}=G_{1}^{*} \circ G_{2}^{*}=(V, E)$ where $V=V_{1} \times V_{2}$ and $E=$ $\left\{\left(\left(u, u_{2}\right),\left(u, v_{2}\right)\right): u \in V_{1},\left(u_{2}, v_{2}\right) \in E_{2}\right\} \bigcup\left\{\left(\left(u_{1}, w\right),\left(v_{1}, w\right)\right):,\left(u_{1}, v_{1}\right) \in E_{1}, w \in\right.$ $\left.V_{2}\right\} \cup\left\{\left(\left(u_{1}, u_{2}\right),\left(v_{1}, v_{2}\right)\right):\left(u_{1}, v_{1}\right) \in E_{1}, u_{2} \neq v_{2}\right\}$. Then the composition of two fuzzy graphs $G_{1}$ and $G_{2}$ is a fuzzy graph $G=G_{1} \circ G_{2}:\left(\sigma_{1} \circ \sigma_{2}, \mu_{1} \circ \mu_{2}\right)$ defined by

$$
\left(\sigma_{1} \circ \sigma_{2}\right)\left(u_{1}, u_{2}\right)=\left(\sigma_{1}\left(u_{1}\right) \wedge \sigma_{2}\right)\left(u_{2}\right), \forall\left(u_{1}, u_{2}\right) \in V
$$

and $\left(\mu_{1} \circ \mu_{2}\right)\left(\left(u, u_{2}\right),\left(u, v_{2}\right)\right)=\sigma_{1}(u) \wedge \mu_{2}\left(u_{2}, v_{2}\right) \forall u \in V_{1},\left(u_{2}, v_{2}\right) \in E_{2}$

$$
\left(\mu_{1} \circ \mu_{2}\right)\left(\left(u_{1}, w\right),\left(v_{1}, w\right)\right)=\sigma_{2}(w) \wedge \mu_{1}\left(u_{1}, v_{1}\right) \forall w \in V_{2},\left(u_{1}, v_{1}\right) \in E_{1}
$$

$\left(\mu_{1} \circ \mu_{2}\right)\left(\left(u_{1}, u_{2}\right),\left(v_{1}, v_{2}\right)\right)=\sigma_{2}\left(u_{2}\right) \wedge \sigma_{2}\left(v_{2}\right) \wedge \mu_{1}\left(u_{1}, v_{1}\right) \quad \forall\left(\left(u_{1}, u_{2}\right),\left(v_{1}, v_{2}\right)\right) \in$ $E-E^{*}$

where $E^{*}=\left\{\left(\left(u, u_{2}\right),\left(u, v_{2}\right)\right): u \in V_{1},\left(u_{2}, v_{2}\right) \in E_{2}\right\} \cup \quad\left\{\left(\left(u_{1}, w\right),\left(v_{1}, w\right)\right): w \in\right.$ $\left.V_{2},\left(u_{1}, v_{1}\right) \in E_{1}\right\}$

Proposition 25. Let $G_{1}:\left(\sigma_{1}, \mu_{1}\right)$ and $G_{2}:\left(\sigma_{2}, \mu_{2}\right)$ be two fuzzy graphs with $G_{1}^{*}:\left(V_{1}, E_{1}\right)$ and $G_{2}^{*}:\left(V_{2}, E_{2}\right)$ with $V_{1} \cap V_{2}=\phi$. Then $\operatorname{CONN}_{\mathrm{G}_{1} \circ \mathrm{G}_{2}}\left(\left(\mathrm{u}_{1}, \mathrm{u}_{2}\right),\left(\mathrm{v}_{1}, \mathrm{v}_{2}\right)\right) \leq$

$\wedge\left\{\operatorname{CONN}_{\mathrm{G}_{1}}\left(\mathrm{u}_{1}, \mathrm{v}_{1}\right), \operatorname{CONN}_{\mathrm{G}_{2}}\left(\mathrm{u}_{2}, \mathrm{v}_{2}\right)\right\}, \forall \mathrm{u}_{1}, \mathrm{v}_{1} \in \mathrm{V}_{1}, \mathrm{u}_{2}, \mathrm{v}_{2} \in \mathrm{V}_{2}$ 
Strong Domination Parameters in Operations on Fuzzy Graphs

Proof: By definition of connectivity of fuzzy graphs[14] $1,2,3 \cdots\}$

$$
\operatorname{CONN}_{G_{1} \circ G_{2}}\left(\left(u_{1}, u_{2}\right),\left(v_{1}, v_{2}\right)\right)=\sup \left\{\left(\mu_{1} \circ \mu_{2}\right)_{k}\left(\left(u_{1}, u_{2}\right),\left(v_{1}, v_{2}\right)\right): k=\right.
$$

Since $\sup (f \circ g) \leq \Lambda \quad\{\operatorname{supf}, \operatorname{supg}\}$ the result follows.

Theorem 26. Let $D_{1}$ and $D_{2}$ be strong dominating sets of the fuzzy graphs $G_{1}:\left(\sigma_{1}, \mu_{1}\right)$ and $G_{2}:\left(\sigma_{2}, \mu_{2}\right)$ respectively. Then $D_{1} \times D_{2}$ is a strong dominating set of $G_{1} \circ G_{2}$.

Proof: We have to prove that for every arc $(u, v) \in\left(V_{1} \times V_{2}\right)-\left(D_{1} \times D_{2}\right)$ there exists an $\operatorname{arc}\left(u^{\prime}, v^{\prime}\right)$ such that $\left((u, v),\left(u^{\prime}, v^{\prime}\right)\right.$ is a strong arc. Let $(u, v) \in\left(V_{1} \times V_{2}\right)-\left(D_{1} \times\right.$ $D_{2}$ ). Then $u \notin D_{1}$ or $v \notin D_{2}$

CASE 1: $u \notin D_{1}$ and $v \in D_{2}$.

Since $D_{1}$ is a strong dominating set of $G_{1}$ there exists a node $u_{1} \in D_{1}$ which strongly dominates $u$. That is, $\left(u, u_{1}\right)$ is a strong arc. Hence $\operatorname{CONN}_{G_{1}}\left(u, u_{1}\right)=\mu_{1}\left(u, u_{1}\right)$

Now, $\left(u_{1}, v\right) \in D_{1} \times D_{2}$ and

$$
\begin{aligned}
&\left(\mu_{1} \circ \mu_{2}\right)\left((u, v),\left(u_{1}, v\right)\right)=\sigma_{2}(v) \wedge \quad \mu_{1}\left(u, u_{1}\right) \\
&=\sigma_{2}(v) \wedge \operatorname{CONN}_{G_{1}}\left(u, u_{1}\right) \\
& \geq \operatorname{CONN}_{G_{2}}\left(v, v_{i}\right) \wedge \operatorname{CONN}_{G_{1}}\left(u, u_{1}\right) \forall v_{i} \in V_{2} \\
& \geq \operatorname{CONN}_{G_{1} \circ G_{2}}\left((u, v),\left(u_{1}, v\right)\right)
\end{aligned}
$$

Hence $\left((u, v),\left(u_{1}, v\right)\right)$ is a strong arc. Hence $\left(u_{1}, v\right)$ strongly dominates $(u, v)$ in $G_{1} \circ G_{2}$.

CASE II: $u \in D_{1}$ and $v \notin D_{2}$.

Since $D_{2}$ is a strong dominating set of $G_{2}$ there exists a node $v_{2} \in D_{2}$ which strongly dominates $v$. That is, $\left(v, v_{2}\right)$ is a strong arc. Hence $\operatorname{CONN}_{G_{2}}\left(v, v_{2}\right)=\mu_{2}\left(v, v_{2}\right)$

Now, $\left(u, v_{2}\right) \in D_{1} \times D_{2}$ and

$$
\begin{aligned}
&\left(\mu_{1} \circ \mu_{2}\right)\left((u, v),\left(u, v_{2}\right)\right)=\sigma_{1}(u) \wedge \mu_{2}\left(v, v_{2}\right) \\
&=\sigma_{1}(u) \wedge \operatorname{CONN}_{G_{2}}\left(v, v_{2}\right) \\
& \geq \operatorname{CONN}_{G_{1}}\left(u, u_{i}\right) \wedge \operatorname{CONN}_{G_{2}}\left(v, v_{2}\right) \forall u_{i} \in V_{1} \\
& \geq \operatorname{CONN}_{G_{1} \circ G_{2}}\left((u, v),\left(u, v_{2}\right)\right)
\end{aligned}
$$

Hence $\left((u, v),\left(u, v_{2}\right)\right)$ is a strong arc. Hence $\left(u, v_{2}\right)$ strongly dominates $(u, v)$ in $G_{1} \circ G_{2}$.

CASE III: $u \notin D_{1}$ and $v \notin D_{2}$.

Since $D_{1}$ and $D_{2}$ are strong dominating sets of $G_{1}$ and $G_{2}$ respectively, there exist nodes $u_{1} \in D_{1}$ and $v_{2} \in D_{2}$ such that $u_{1}$ strongly dominates $u$ in $G_{1}$ and $v_{2}$ strongly dominates $v$ in $G_{2}$.

Then $\operatorname{CONN}_{G_{1}}\left(u, u_{1}\right)=\mu_{1}\left(u, u_{1}\right)$ and $\operatorname{CONN}_{G_{2}}\left(v, v_{2}\right)=\mu_{2}\left(v, v_{2}\right)$

Now $\left(u_{1}, v_{2}\right) \in D_{1} \times D_{2}$ and

$$
\begin{gathered}
\left(\mu_{1} \circ \mu_{2}\right)\left((u, v),\left(u_{1}, v_{2}\right)\right)=\sigma_{2}(v) \wedge \sigma_{2}\left(v_{2}\right) \wedge \mu_{1}\left(u, u_{1}\right) \\
\geq \mu_{2}\left(v, v_{2}\right) \geq \wedge \quad \operatorname{CONN}_{G_{1}}\left(u, u_{1}\right) \\
=\operatorname{CONN}_{G_{2}}\left(v, v_{2}\right) \wedge \quad \operatorname{CONN}_{G_{1}}\left(u, u_{1}\right) \\
\geq \operatorname{CONN}_{G_{1} \circ G_{2}}\left((u, v),\left(u_{1}, v_{2}\right)\right)
\end{gathered}
$$

Hence $\left((u, v),\left(u_{1}, v_{2}\right)\right)$ is a strong arc. Hence $\left(u_{1}, v_{2}\right)$ strongly dominates $(u, v)$ in $G_{1} \circ G_{2}$.

Hence $D_{1} \times D_{2}$ is a strong dominating set of $G_{1} \circ G_{2}$. 


\section{O.T.Manjusha}

\section{Conclusion}

In this paper, the concept of domination is introduced on various operations on fuzzy graphs such as union,join, composition and cartesian product using strong arcs. The different types of strong domination parameters of union and join of two fuzzy graphs is obtained.It is obtained the strong domination set of cartesian product of two fuzzy graphs.

Acknowledgement. I am very grateful to the reviewer for the comments and the suggestions towards improving this article.

\section{REFERENCES}

1. K.R.Bhutani, On automorphisms of fuzzy graphs, Pattern Recognition Letters, 9 (1989) 159-162.

2. K.R.Bhutani and A.Rosenfeld, Strong arcs in fuzzy graphs, Inform. Sci., 152 (2003) 319-322.

3. K.R.Bhutani and A.Rosenfeld, Fuzzy end nodes in fuzzy graphs, Inform. Sci., 152 (2003) 323-326.

4. K.R.Bhutani and A.Batton, On M-strong fuzzy graphs, Inform. Sci., 1559 (2003) 103-109.

5. G.Chartrand and P.Zang, Introduction to graph theory, Tata McGraw-Hill Edition 2006.

6. T.W.Haynes, S.T.Hedetniemi and P.J.Slater, Fundamentals of domination in graphs, Marcel Dekker, Inc., New York 1998.

7. O.T.Manjusha and M.S.Sunitha, Notes on domination in fuzzy graphs, Journal of intelligent and Fuzzy Systems, 27 (2014) 3205-3212.

8. O.T.Manjusha and M.S.Sunitha, Total domination in fuzzy graphs using strong arcs, Annals of Pure and Applied Mathematics, 9(1) (2014) 23-33.

9. O.T.Manjusha and M.S.Sunitha, Strong domination in fuzzy graphs, Fuzzy Inf. Eng., 7 (2015) 369-377.

10. O.T.Manjusha and M.S.Sunitha, Connected domination in fuzzy graphs using strong arcs, Annals of Fuzzy Mathematics and Informatics, Accepted.

11. O.T.Manjusha and M.S.Sunitha, Independent domination in fuzzy graphs using strong arcs, Communicated.

12. O.T.Manjusha and M.S.Sunitha, Coverings, matchings and paired domination in fuzzy graphs using strong arcs, Iranian Journal of Fuzzy Systems, 16(1) (2019) 145-157

13. J.N.Mordeson and P.C.-Shyh, Operations on fuzzy graphs, Inform. Sci., 79 (1994) 159-170.

14. J.N.Mordeson and P.S.Nair, Fuzzy graphs and fuzzy hypergraphs, Physica - Verlag 2000.

15. A.Nagoorgani and V.T.Chandrasekaran, Domination in fuzzy graph, Adv. in Fuzzy Sets and Systems, 1(1) (2006) 17-26.

16. A.Nagoorgani and P.Vijayalakshmi, Insensitive arc in domination of fuzzy graph, Int. J. Contemp. Math. Sciences, 6(26) (2011) 1303-1309.

17. A.Nagoor Gani and P.Vadivel, A study on domination, independent domination and irredundance in fuzzy graph, Applied Mathematical Sciences, 5(47) (2011) 2317 2325.

18. P. Debnath, Domination in interval-valued fuzzy graphs, Ann. Fuzzy Math. Inform., 
Strong Domination Parameters in Operations on Fuzzy Graphs

6(2) (2013) 363-370.

19. A.Rosenfeld, Fuzzy graphs, in fuzzy sets and their application to cognitive and decision processes, Academic Press, (1975) 77-95.

20. E.Sampathkumar and H.B.Walikar, The connected domination number of a graph, Jour. Math. Phy. Sci., 13(6) (1979) 607-613.

21. K.R.Sandeep Narayan and M.S.Sunitha, Connectivity in a fuzzy graph and its complement, Gen. Math. Notes, 9(1) (2012) 38-43.

22. A.Somasundaram and S.Somasundaram, Domination in fuzzy graphs-I, Pattern Recognition Letters, 19 (1998) 787-791.

23. A.Somasundaram, Domination in fuzzy graphs-II, J. Fuzzy Math., 13 (2) (2005) 281-288.

24. A.Somasundaram, Domination in products of fuzzy graphs, Inter. Journal of Uncertainty Fuzziness and Knowledge-Based Systems, 2 (2005) 195-204.

25. Sunil Mathew and M.S.Sunitha, Types of arcs in a fuzzy graph, Inform. Sci., 179 (2009) 1760-1768.

26. S. Mathew and M.S.Sunitha, Node connectivity and arc connectivity of a fuzzy graph, Inform. Sci., 180 (2010) 519-531.

27. M.S.Sunitha and A.Vijayakumar, Complement of a fuzzy graph, Indian Journal of Pure and Applied Mathematics, 33(9) (2002) 1451-1464.

28. M.S.Sunitha and A.Vijayakumar, A characterization of fuzzy trees, Inform. Sci., 113 (1999) 293-300.

29. A.A.Talebi and H.Rashmanlou, Isomorphism on interval valued fuzzy graphs, Ann. Fuzzy Math. Inform, 6(1) (2013) 47-58.

30. R.T.Yeh and S.Y.Bang, Fuzzy relations, fuzzy graphs and their applications to clustering analysis, in: L.A. Zadeh, K.S. Fu, M. Shimura (Eds.), Fuzzy sets and Their Applications, Academic Press (1975) 125-149.

31. M.Cary, Perfectly regular and perfectly edge-regular fuzzy graphs, Annals of Pure and Applied Mathematics, 16(2) (2018) 461-469.

32. S.Mathew and M.S.Sunitha, Fuzzy graph theory: a survey, Annals of Pure and Applied Mathematics, 4(1) (2013) 92-110.

33. T.Pathinadhan and J.Jesintha, Matrix representation of double layered fuzzy graph its properties, Annals of Pure and Applied Mathematics, 8(2) (2014) 51-58. 